\title{
Lusioersily
}

\section{Development and psychometric testing of the physical examination attitudes and Practices Scale}

Gharaibeh, B., Al-Smadi, A. M., Ashour, A., \& Slater, P. F. (2019). Development and psychometric testing of the physical examination attitudes and Practices Scale. Nursing Forum, 54(1), 111-120. https://doi.org/10.1111/nuf.12304

Link to publication record in Ulster University Research Portal

\section{Published in:}

Nursing Forum

Publication Status:

Published (in print/issue): 10/02/2019

DOI:

10.1111/nuf.12304

Document Version

Author Accepted version

\section{General rights}

Copyright for the publications made accessible via Ulster University's Research Portal is retained by the author(s) and / or other copyright owners and it is a condition of accessing these publications that users recognise and abide by the legal requirements associated with these rights.

\section{Take down policy}

The Research Portal is Ulster University's institutional repository that provides access to Ulster's research outputs. Every effort has been made to ensure that content in the Research Portal does not infringe any person's rights, or applicable UK laws. If you discover content in the Research Portal that you believe breaches copyright or violates any law, please contact pure-support@ulster.ac.uk. 


\section{NURSING \\ FOrUM an independent voice for nursing}

\section{Development and psychometric testing of the physical examination attitudes and Practices Scale}

\begin{tabular}{|c|c|}
\hline Journal: & Nursing Forum \\
\hline Manuscript ID & NF-11-17-OA-0886.R3 \\
\hline Manuscript Type: & Original Article \\
\hline Search Terms: & factor analysis, physical exam, Nursing Research \\
\hline
\end{tabular}




\title{
Development and psychometric testing of the Physical Examination Attitudes and Practices Scale
}

\begin{abstract}
Aim:

To develop and test a scale that assesses the attitudes and practices of registered nurses toward physical examination in of (delete) the clinical settings.

Methods:

A cross sectional methodological design with a convenience sample of 277 registered nurses was used. A likert-type scale was constructed using 55 likert-type items that were extracted from relevant literature. Exploratory Factor Analyses were conducted using Varimax rotation. Factor loading, Eigenvalues and screeplots were used to determine the best fit model.

Results:

The final version of the scale consisted of four factors. The determinant score was $(0.001)$ and the total variance explained was $56.26 \%$. All of those 4 factors had Eigenvalue $>1$. The final version of the scale (the 20- item scale) was tested for reliability and was internally consistent (Cronbach's alpha $=.833)$. The scale was supported for its validity.

\section{Conclusion:}

The final version of the scale can be used to determine nurses' perspective and use of physical examination, and assist in bridging the gap between what is taught and what is practices regarding Physical Examination.
\end{abstract}

Keywords: factor analysis, physical examination, registered nurses, attitudes, practices, barriers to physical examination. 


\section{Development and psychometric testing of the physical examination attitudes and Practices \\ Scale}

\section{Introduction}

Physical Examination (PE) is an integral part of patient care that enables health care professionals, including nurses, to collect and analyze data based on scientific methods (Jarvis, 2004; Rhoads \& Peterson, 2014). Physical examination is an essential skill in nursing practice and is therefore an essential component in nursing education. It helps in providing holistic nursing care, and strengthens nurse-patient relationship and is a recognized skill at both national and international level (Giddens, 2007, Jordanian Nursing Council, 2015). Some believe that PE in its modern form started in 1761 and has continued to evolve ever since (Verghese, Charlton, Cotter, \&Kugler, 2011).

The rapidly developing health care systems, the presence of new technologies that undermines the need for examination techniques, and an overwhelmed health system are forcing health care professionals to rely less of PE and depend more on the gold standards of diagnosing (Brown \& Lemery, 2009), such as imagining study Verghese (2011). Verghese (2011) indicated that patients usually have an imaging study even before being seen by a physician and having the results of such a reliable test, the physician then perceived little importance or necessity of conducting PE.

Zambas (2010) indicated that the lack of confidence in PE among nurses is a main reason for the disconnection between what is taught and what is practiced. This disconnection might be the reason why a substantial percentage of PE skills are never used in the clinical setting. The lack of clarity of the usefulness of PE made some nurses call into question the relevance of PE in nursing practice. So, (delete) Zambas (2010) indicated that by addressing what the nurses 
recognize and perceive about PE are important because understanding their attitudes toward PE can help in bridging the gap between what is taught and what is practiced.

Therefore, the assessment of nurse's attitudes toward PE is important especially as the use of PE in practice very much depends on how nurses view its relevance in the caring process (Aldridge-Bent, 2011). Most studies in the literature tend to focus on the relative contribution of PE in diagnosis, or assess the frequency of performing certain skill (Giddens, 2007; Secrest, Norwood, \&duMont, 2005; Melaniea, Robynb, Ainsleyc, Catherinec, Jenny, 2013) . However, there is lack of research that investigated actual health assessment practices and factors affecting the performance of PE in the clinical areas (Lillibridge \& Wilson, 1999; McElhinney, 2010).

Muhrer (2014) emphasized the need to investigate whether PE skills were being used in the clinical setting, assessing how frequent $\mathrm{PE}$ is performed, and identifying the barriers that hinder performing PE. In addition, Aldridge-Bent (2011) emphasized the need to assess nurse's attitudes toward PE. However, there are lack of studies that investigate actual health assessment practices and factors affecting the performance of PE in the clinical areas (Lillibridge \& Wilson, 1999; McElhinney, 2010).

\section{Literature Review}

Physical examination can be defined as the assessment done by the application of the classical skills of inspection, palpation, percussion, and auscultation (Schroyen, George, Hylton, \& Scobie, 2005). Verghese, Charlton, Cotter, and Kugler (2011) indicated that there has been controversy about the actual usefulness of PE, which prompted a number of studies being conducted to evaluate the importance of PE in diagnosing and treating the patients.

With the expanding role of nursing, the educational needs for the nurses should be revised to insure proper focused education (Edmunds, Ward, \& Barnes, 2010). This role 
expansion, the rapidly developing health care system, and the ever-growing challenges nurses are facing, created concerns that nursing graduates are not adequately prepared to enter practice (Giddens, 2007). Some educationalists noted that nurses need to broaden their health assessment knowledge (Lesa \& Dixon, 2007); while others indicated that a large proportion of the assessment skills that nursing student are taught are not used in or are irrelevant to the clinical practice (Giddens, 2007). This disagreement stimulated research into what factors influenced the implementation of physical assessment into the nurses' assessment (Secrest, Norwood, \&duMont, 2005).

Hampton, Harrison, Mitchell, Prichard, Carol, and Seymour (1975) argued that PE is sought as a resource to identify patient's problems and make medical diagnosis, however, there is no agreement about how useful eliciting physical signs to identify the patient's problems. In fact, the authors explicitly stated that no final diagnosis can be done with certainty without doing detailed laboratory testing. They conducted a study using a sample of 80 patients to assess the relative contribution of history- taking, PE, and laboratory testing to diagnose the conditions. Physical examination was found to be useful in the diagnosis of only seven patients.

More recently, Kugler and Verghese (2010) listed four reasons to continue using PE.(1), many diagnoses can be readily made by using PE, (2), PE is a good tool to generate hypotheses regarding the diagnosis, (3), PE strengthen the patient- healthcare provider relationship, finally, (4) knowing PE is required to pass the examinations and acquire the degree. Kugler and Verghese (2010) indicated that failure of the PE procedure to diagnose a problem might be due to the fact that the health care professionals identify themselves doing the PE the right way while actually they do not; this might be attributed to inconsistency in the way PE is done and to the existing doubt about the reliability of the PE findings. 
Verghese, Charlton, Cotter, and Kugler (2011) argued that PE should be viewed as a ritual and essential part of patient care even if it is poorly reliable or reproducible. This poor reliability of the PE might be less useful when working on atypical patient. They identified the expansion of use of imaging studies as an important reason for undermining PE. Furthermore, they indicated that patients usually have an imaging study even before being seen by a physician. Having the results of a reliable testing, the physician then perceived little importance of conducting PE; which weakens the physician-patient relationship.

There is disagreement about the usefulness of PE in the medical field regarding largely because of the lack of reliability of PE in making medical diagnosis; and in being source of diagnostic error (Benbassat, 2015). In nursing, the controversy takes different approaches where some researchers argued that a PE is not the (delete) responsibility of nurses because diagnosing the problem is the physician's responsibility (Zambas, 2010). Other studies disagreed and emphasized that PE is an important part of a holistic approach to nursing care (Birks et al.,2014) and that PE is an essential skill to learn because nurses role is expanding (Aldridge-Bent, 2011). This controversy stimulated research in what PE skills are useful and frequently done versus what skills are seldom done. For example, Birks et al., (2014) who found that about third of the listed skills were reported as frequently done. The researcher raised questions about the required depth of conducting and teaching PE to nurses.

Several factors where identifies in the literature as possible obstacles for performing PE. For example, Douglas, et al., (2014) indicated that it is responsibility of physician to conduct a PE and its inappropriateness to clinical setting topped the list of these obstacles. Other factors included nurses confidence (Schroyen, et al., 2005), substituting PE with imaging studies (Verghese et al., 2011), rule confusion (Zambas, 2010), and educational factors, lack of 
resources (Aldridge-Bent, 2011). Moreover, McElhinney, (2010) conducted a Delphi study with nurses to identify factors that help or hinder the performance of PE skills on the clinical area. They aggregated these factors into four main themes which were individual factors (e.g. individual self $\square$ confidence), organizational factors (e.g. role clarity), educational factors (e.g. effective educational preparation), and support of others (e.g. support from other disciplines).

Despite that many barriers in conducting a PE reported in the literature, there is still a need to continue asking questions regarding whether physical assessment skills were being utilized in the clinical setting, how frequently is PE performed, and whether the barriers to conduct PE do exist (Muhrer, 2014).

In summary, there are more calls to more incorporation of PE skills by the nurses because nursing role is expanding. However, these calls are met with opposing forces that questions the depth of PE skills need to be taught to nurses because many of these skills are never used in practice. Several factors were identified and seemed to influence the performing of PE in the clinical settings. So, (delete) This study was to develop and test a scale that assesses the attitudes and practices of registered nurses toward PE of in the clinical settings.

\section{Methods}

A cross-sectional, descriptive design was used to: assess the registered nurses' attitudes and practices toward PE the clinical setting.

\section{Participants}

A convenience sampling technique was used to recruit registered nurses from five hospitals. The inclusion criteria were; registered nurses that hold diploma degree or higher, working in clinical area, and welling to participate in the study. Sample size was calculated using G* power software (Faul, Erdfelder, Lang \& Buchner 2007). The sample size calculation was 
based on the use of F-Test and a medium effect size is required for the purpose of this study which was (.15). The sample size was determined according to the power level which was 0.80 and the use of conventional $\alpha=.05$ two tailed criterion of the significance. Based on the sample size determination technique, a sample of at least 184 nurses was required for this study.

\section{Setting}

This study was conducted in five hospitals in North of Jordan. These hospitals are governmental (public) hospitals and considered central hospital where which health care services are provided to all patients regardless of their residential area. Nurses from various hospital units were recruited such as medical- surgical, ICU, CCU, OR, ER, pediatric and maternity units, and outpatient clinics.

\section{Development of the Scale}

Following an extensive search of the relevant literature, a likert- type scale was constructed using 55 items that were extracted from the reviewed articles (see appendix 1). These items were developed based on the literature and previous studies that examined attitudes and practices toward PE, and addressed possible barriers to practicing PE by health care professionals. The likert- type scale used answers that ranged from (1) totally disagree; (2) disagree; (3) neutral/ neither agree nor disagree; (4) agree; and (5) totally agree. A demographic sheet was also added to the questionnaire. The items of the scale assessed attitudes and practices toward PE, and assessed how often the nurses conducted PE. This scale was examined for face validity, clarity, and agreement about the denotation of the items by an expert panel of three PhD- prepared nurses who teach Health Assessment classes in Schools of Nursing from different universities in Jordan. Then, the scale was translated to Arabic language. The scale was pilot tested before using it in the main study using $10 \mathrm{RNs}$. The comments from the $\mathrm{PhD}$ expert panel 
and the RNs resulted in minor amendments such as language editing of the items so that the final items are easily and consistently understood. The total attitude and practices toward performing PE was calculated by summing the individual responses on each item after reverse code the items that were positively worded, so that all items are negatively stated. The final score can range from 55 to 275 . The higher the score, the lower level of attitudes the nurses have regarding performing of PE in the clinical setting. The reliability of the 55- item scale was tested using the internal consistency coefficient (Cronbach's alpha= .924).

\section{Data Analysis}

SPSS 22 was used to analyze the data. Descriptive statistics that included mean, SD, range, and frequency was used to describe sample characteristics. Kaiser-Meyer-Olkin (KMO) Measure of Sampling Adequacy and Bartlett's Test of Sphericity were done to assess the suitability of the data for factor analysis. Exploratory factor analysis technique was used with Principal Component Analysis to identify factors, refine items, , and assess the psychometric properties of the scale. Correlation matrices were checked to identify the type of rotation (oblique or orthogonal). Adequate sample size, suitability of data for factor analysis, and absence of multicollinearity are measured using a KMO measure of 0.50 or more, a significant Bartlett's Test of Sphericity, and a determinant score that is greater than 0.00001 respectively (Hair et al, 2006; Williams et al., 2010).

Exploratory Factor Analysis with rotated matrix (Varimax rotation) was used. The acceptable factor loading for each item was 0.5 or higher (Hair et al, 2006). This level of item factor loading was selected to ensure a robust analysis, and strong factor model as this was the first factor analysis conducted on the scale. Exploratory factor analysis was repeated after the removal of the items that failed to load onto a factor removing the lower loaded items. This 
process was repeated until all items had factor loading of 0.5 or higher. Then, Eigenvalue and the scree plot were used to determine the number of factors to be retained (Yong \& Pearce, 2013). After determining the number of factors to be retained, the analyses were re- run where the number of factors extracted was manually set until the analysis provides the most desirable factor structure (Costello \& Osborne, 2005).

Factor analysis was conducted in two stages. In stage one, a series of principal component analyses were done whereby items that had low item loading were removed and the analysis repeated. These steps were repeated until the final analysis contained a scale with all the items have acceptable item loading. In stage two, analyses were done to identify the best number of factors that constitute the scale. Stage two contained two phases. In phase one, scree plots and Eigenvalues were examined. In phase two, the analyses were done where which the number of factors (as identified in the previous phase) was set manually until the analysis provides the most desirable rotated factor structure.

\section{Data Collection Procedure}

Human Subject Committee approval was obtained from the Institutional Review Board of the University prior to data collection. Research assistants (RAs) were trained for data collection. The RAs are instructed to approach the potential participants in their clinical settings. The RAs provided a brief description of the study and invited the nurses to participate. The RNs, who agree to participate, received a questionnaire package containing a cover letter outlining a summary of the study, the participant's rights regarding refusal to participate, and the researcher's contact information. The cover letter also encouraged the participants to complete the questionnaire and returned it as soon as possible to the RAs. 


\section{Ethical Issues}

The study method and protocol were reviewed and approved by the Faculty of Nursing Research Committee for Protection of Human Subjects and by the institutional review board. Written informed consent was obtained from all the participants in the study. The participants received both verbal and written information about the purpose; content and extent of the study. The confidentiality of the participants was protected by providing code number for each participant at the stage of data collection and analysis. In addition, the collected questionnaires were kept in a locked cabinet to keep the participants information private and confidential. Participants' participation was completely voluntary and they were assured that their responses will be confidential. All questionnaires were disposed off after study completed.

The participants were informed that they had the right to withdraw from the study at any time without any effect on their achievement. No risk affected the participants, since the data collection process mainly relies on a descriptive questionnaire.

\section{Results}

\section{Sample characteristics}

A convenience sample of $n=277$ nurses participated in the study (See Table 1 for sample characteristics). About quarter of the participants ( $n=70 ; 25.3 \%)$ were from ICU or CCU units. Those who worked in acute care units (i.e. ER, recovery room, OR; n=44) composed about $15.8 \%$ of the sample. The remaining participants were from ordinary floors and outpatient clinics. See Table 1 for descriptive statistics about sample characteristics. About three quarters of the sample had a Bachelor degree in nursing and only a small percent of less than $1 \%$ of the sample had a post graduate degree in nursing $(n=15)$. 
[Insert table 1 here]

\section{Psychometric testing of the scale}

Exploratory factor analysis of the scale was conducted using Principal Component Analysis with Varimax Rotation. The orthogonal rotation (Varimax) was selected because the items were not highly correlated with each other (The highest item to item correlation was 0.69 , less than 0.8). Prior to the extraction of the factors, Kaiser-Meyer-Olkin (KMO) Measure of Sampling Adequacy and Bartlett's Test of Sphericity were done to assess the suitability of the data for factor analysis. The result of the KMO measure showed that the sample size is sufficient to conduct factor analysis (Kaiser-Meyer-Olkin Measure of Sampling Adequacy= .873). Moreover, Bartlett's Test of Sphericity was statistically significant $(p=.001)$ indicating that the factor analysis is suitable.

In stage one, factor extraction was done using the rotated matrix where item loading was checked, and the items that had unacceptable item loading (less than 0.5) were removed. After three rounds of analyses, a total of 26 items were removed and the remaining 29 items were kept. The results showed that the determinant score in the last round was $2.28 \mathrm{E}-5$ indicating absence of multicolinearity. KMO measure of sampling adequacy was .862 and Bartlett's Test of Sphericity was statistically significant $(\mathrm{p}=.001)$ indicating that the factor analysis is suitable.

In stage two, the analysis showed that the 29 items, that were kept from stage one, loaded into 8 components. However, the scree plot and the eigenvalues showed that the data is likely to have 3 or 4 underlying factors (See figure 1 for the scree plot and Table 2 for the rotated matrix). So, principal component analyses were iteratively redone where a fixed number of factors were selected. In phase one, and using the retained 29 items from the previous analysis, the fixed 
number of factors was set at 3 . Two rounds of analyses were done where nine items that had poor item loading $(<.5)$ were removed. The final analysis included 20 items. This 20 - item solution had a determinant score of $(0.001)$ and accounted for $50.18 \%$ of the variability of the scores. Then, in phase two, and using the same 29 items used at the beginning of phase one, the fixed number of factors was set at 4 . The 4 factor solution was tested across 2 rounds of analyses. In the second round, a total of 20 items were retained. The determinant score was 0.001 and the total variance explained was 56.26\%. All 4 factors had Eigenvalue $>1$. The final version of the scale (the 20- item scale) was tested for reliability and was internally consistent (Cronbach's alpha $=.833$ ). An acceptable value of Cronbach's alpha ranges from 0.70 to 0.95 (Tavakol \& Dennick, 2011).

[Insert figure 1 here]

[Insert table 2 here]

The four factors that resulted from the final analysis were qualitatively assessed for the purpose of naming these factors (See table 3 that shows the factors that composed the final version of the scale). Further assessment of the psychometric properties of the new 20- item scale was done (See Table 4 for the descriptive statistics of the 20 items that composed the scale). The correlation coefficient Pearson $r$ between the original 55 item scale and the new 20 item scale was done and showed that the two measures were strongly correlated $(r=.913 ; \mathrm{p}<.001)$. This results support the validity of the new 20 item scale. The internal consistency reliability of the 20- item scale and the internal consistency reliability of each of the subscales were acceptable and above the .7 level. Only the last factor, (cultural difficulties), had an internal reliability that was below the acceptable level. However, this factor had only 3 items. Tavakol and Dennick 
(2011) stated that Cronbach's alpha is affected by the number of the items that composes it and the value of alpha is reduced if the scale is too short.

[Insert table 3 here]

[Insert table 4 here]

\section{Discussion}

The attitude and practice toward PE scale was developed specifically to explore the attitudes and the practices of the RNs toward the use of PE. This scale can be used to address the RNs views regarding PE and the possible barriers that may hinder the application and performing PE. The study resulted in developing a simple, valid, reliable, and relatively short questionnaire that contains 20 items to assess how the RNs in the clinical setting perceive PE. This assessment is necessary because embracing and performing PE in practice depends on how nurses view its relevance (Aldridge-Bent, 2011).

The factor analysis of the scale resulted in four factors solution. These four factors were identified as 1- technical deficiencies in PE (7 items), 2- Benefits and usefulness ofPE (5 items), 3- Barriers to conduct PE (5 items), and 4- Cultural considerations (3 items). Even though that the forth factor included only three items; this factor was retained because some indicated that three items in a factor is an acceptable solution (Hair, Black, Babin \& Anderson, 2010). Also, this factor is an essential one that has unique contribution to the construct and to the scale. Considering patient's culture is important because it influences how the patients interact with the nurse, and how he understands health concepts (Brega et al., 2015).

The final version of the scale was supported for its reliability and validity. This scale can be useful in addressing the attitudes and practices of the RNs toward PE and identify the areas 
where the RNs may require further focus and support to improve their attitudes to enhance their performing of $\mathrm{PE}$ in the clinical settings. This can contribute into improving various patient outcomes (Aldridge-Bent, 2011; Verghese et al., 2011).

The four factors that were identified by this study were similar to the categories presented described by Kugler and Verghese (2010), who categorized the importance of PE to physicians in four categories (making readily diagnosis, generating hypotheses regarding the diagnosis, strengthening relationship with patient, and passing the examinations to acquire a degree).The factor (benefits and usefulness of PE) identified that nurses consider PE as vital to care despite the given weaknesses and limitations of PE, and recognize the contribution of PE into making a diagnosis. Moreover, this study added further details to those four categories presented by Kugler and Verghese (2010). For example, this study showed that PE is not only essential to make diagnosis, but also considered as a cost efficient way to do so by limiting the un-necessary laboratory and imaging testing. In addition, improving the relationship with patients can be attained by enhancing communication and caring opportunities. However, this study differed from Kugler and Verghese's in that passing examinations and obtaining a degree was not recognized by the nurses as an important aspect regarding PE. This finding may indicate that nurses are either more patient- focused, or that the nursing curricula did not provide deep insight about PE techniques and focused on general content. Further investigation in this regard is needed.

The items of the scale represent issues and barriers that RNs may face in their practice that can influence their performance of PE. Even though the items that represent these issues were studied in a sample of nurses, these issues and barriers are also thought not only to affect nurses but also other health care professionals. Thus, this scale can be useful to assess the 
attitudes and practices of other health care professional toward PE. However, further research regarding the suitability of this scale to other professions is needed. These futuristic researches that use this scale can also be helpful in detecting the differences between the various health care professions with regard to the importance of performing PE in their clinical settings, the attitudes and practices of their members toward PE, and the importance and the depth of integrating PE into their educational curricula or their training classes. These studies might assist in improving the attitudes and practices toward PE in a way that reduces the dependence on diagnostic and imaging studies (Brown \& Lemery, 2009).

Finally, several barriers that are thought to hinder performing PE were listed in this new instrument. So, this instrument can be helpful for the different clinical settings to identify the individualized needs of their nurses. In other words, this instrument can help in identifying if certain nurses or groups of nurses or certain nurses in certain units need further education and support regarding performing PE. Identifying the individualized educational needs and need for support can help in creating efficient programs to support and enhance the nurses perspectives and implementation of PE techniques for their patients. These efficient programs could assist in bridging the gap between what is taught and what is practiced and improve nurses' confidence in performing PE (Zambas, 2010).

\section{Conclusion and Implication}

Physical Examinations are considered an essential part of comprehensive patient care, nurses should perceive it as such. Thus assessing nurses' attitudes and practices toward PE is important because the way they view PE can influence performing it in the clinical setting. Various factors were addressed by the literature that may hinder this performance. So, this study examined the psychometric properties of a scale that was developed for the purpose of 
examining the nurses' view using items that were extracted from relevant literature. The final version of the scale was supported for its reliability and validity. The final version of the scale consisted of 20 items that were fit into four different factors. This scale can be used in clinical and research settings.

\section{Limitations}

The study was conducted primarily on registered nurses, so the use of the scale on members of other health care professions might be restricted and may introduce threat to the internal validity of the scale, and because other professions may encompass different perspective toward PE than that of the nurses. In addition, the forth factor of the scale had only three items and a level of Cronbach's alpha below recommended level of 0.7 . This might be due to the small number of items in that factor. The use of convenience sampling technique may introduce threats to the external validity of the scale. Moreover, the testing procedures resulted in reducing the original55- item scale down to a 20 -item scale and resulting in omitting relatively high number of items. The deleted items may have contained important aspects that were removed due to setting the minimum level of item loading at (0.5) instead of using a lower value (e.g. 0.35); or due to the nature of the sample itself. So, further researches are recommended to test the validity of the scale in different professions, and to test the scale in other settings and using different methodology. 


\section{References}

Aldridge-Bent, S. (2011). Advanced physical assessment skills: implementation of a module. British Journal of Community Nursing, 16(2), 84-88.

Benbassat, J. (2015). PE Skills: Learning Difficulties. In TeachingProfessional Attitudes and Basic Clinical Skills to Medical Students. SpringerInternational Publishing.

Birks, M., James, A., Chung, C., Cant, R., \& Davis, J. (2014). The teaching of physical assessment skills in pre-registration nursing programmes in Australia: Issues for nursing education. Collegian, 21(3), 245-253.

Brega AG, Barnard J, Mabachi NM, Weiss BD, DeWalt DA, et al. (2015). AHRQ Health Literacy Universal Precautions Toolkit, Second Edition. (Prepared by Colorado Health Outcomes Program, University of Colorado Anschutz Medical Campus under Contract No. HHSA290200710008, TO\#10.) AHRQ Publication No. 15-0023-EF. Rockville, MD. Agency for Healthcare Research and Quality. January 2015. Available at: https://www.ahrq.gov/sites/default/files/publications/files/healthlittoolkit2_3.pdf

Brown, E. G., \&Lemery, J. (2010). Rescuing the PE. Wilderness \& Environmental Medicine, 21(2), 172-173.

Costello, A.B., \& Osborne, J.W. (2005). Best practices in exploratory factor analysis: Four recommendations for getting the most from your analysis. Practical Assessment, Research and Evaluation, 10(7), 1-9.

Douglas, C., Osborne, S., Reid, C., Batch, M., Hollingdrake, O., \& Gardner, G. (2014). What factors influence nurses' assessment practices? Development of the Barriers to Nurses' use of Physical Assessment Scale. Journal of advanced nursing, 70(11), 2683-2694. 
Edmunds, L., Ward, S., \& Barnes, R. (2010). The use of advanced physical assessment skills by cardiac nurses. British Journal of Nursing, 19(5), 282-287.

Faul, F., Erdfelder, E., Lang AG., Buchner, A. (2007). G*Power 3: a flexible statistical power analysis program for the social, behavioral, and biomedical sciences. Behavior ResearchMethods,39(2):175-91.

Fennessey, A., \&Wittmann $\square$ Price, R. A. (2011). Physical assessment: A continuing need for clarification. Nursing Forum,46( 1), 45-50).

Giddens, J. F. (2007). A survey of physical assessment techniques performed by RNs: lessons for nursing education. Journal of nursing Education, 46(2), 83-87.

Forbes, H., \& Watt, E. (2015).Jarvis's PE and Health Assessment $\left[2^{\text {nd }}\right.$ Ed]. Elsevier Health Sciences.

Hair, J. (2006).Multivariate data analysis. Upper Saddle River, N.J: Pearson Prentice Hall.

Hair, J., Black, W. C., Babin, B. J., \& Anderson, R. E. (2010). Multivariate data analysis (7th ed.). Upper saddle River, New Jersey: Pearson Education International

Jordanian Nursing Council, (2015).Publications. Available at:http://jnc.gov.jo/en/pages/publications.aspx. Accessed April 2017.

Kugler, J., \&Verghese, A. (2010).The physical examination and other forms of fiction. Journal of general internal medicine, 25(8), 756-757.

Lesa, R., \& Dixon, A. (2007). Physical assessment: implications for nurse educators and nursing practice. International Nursing Review, 54(2), 166-172.

McElhinney, E. (2010). Factors which influence nurse practitioners ability to carry out physical examination skills in the clinical area after a degree level module--an electronic Delphi 
study. Journal of Clinical Nursing, 19(21-22), 3177-3187.doi: 10.1111/j.13652702.2010.03304.x

Melaniea, B., Robynb, C., Ainsleyc, J., Catherinec, C., Jenny, D. (2013). The use of physical assessment skills by registered nurses in Australia: Issues for nursing education. Collegian, 20(1),27-33

Muhrer, C. (2014). The importance of the history and physical in diagnosis. The Nurse Practitioner, 39(4), 30-35. doi: 10.1097/01.NPR.0000444648.20444.e6

Osborne, S., Douglas, C., Reid, C., Jones, L., \& Gardner, G. (2015). The primacy of vital signsacute care nurses' and midwives' use of physical assessment skills: a cross sectional study. International Journal of Nursing Studies, 52(5), 951-962.

Schroyen, B., George, N., Hylton, J., \&Scobie, N. (2005). Encouraging nurses' physical assessment skills. Nursing New Zealand, 11(10), 14-15.

Secrest, J. A., Norwood, B. R., \& duMont, P. M. (2005). Physical assessment skills: a descriptive study of what is taught and what is practiced. Journal of Professional Nursing, 21(2), 114-118.

Tavakol, M., \& Dennick, R. (2011). Making sense of Cronbach's alpha. International Journal of Medical Education, 2, 53-55. http://doi.org/10.5116/ijme.4dfb.8dfd

Yong, A. G., \& Pearce, S. (2013). A beginner's guide to factor analysis: Focusing on exploratory factor analysis. Tutorials in quantitative methods for psychology, 9(2), 79-94.

Verghese A, Brady E, Kapur C, Horwitz R. (2011). The Bedside Evaluation: Ritual and Reason. Annals of Internal Medicine, 155(8), 550-553. doi: 10.7326/0003-4819-155-8201110180-00013 
Verghese, A., Charlton, B., Cotter, B., \&Kugler, J. (2011). A history of physical examination texts and the conception of bedside diagnosis. Transactions of the American Clinical and Climatological Association, 122, 290-311.

Williams, B., Onsman, A., \& Brown, T. (December 06, 2010). Exploratory factor analysis: A five-step guide for novices. Journal of Emergency Primary Health Care, 8, 3, 1-13.

Zambas, S. I. (2010). Purpose of the systematic physical assessment in everyday practice: critique of a "sacred cow". Journal of Nursing Education, 49(6), 305-310. doi:10.3928/01484834-20100224-03 
Table 1. Sample characteristics

\begin{tabular}{|c|c|c|c|}
\hline Demographic & Mean (SD) & Range & Frequency (\%) \\
\hline Age & $29.8(5.9)$ & $22-56$ & \\
\hline Experience (years) & $7.3(5.9)$ & Less than 1 year - 34 & \\
\hline $\mathrm{RN}$ to patient ratio & $3.7(2.8)$ & $1-17$ & \\
\hline \multicolumn{4}{|l|}{ Gender } \\
\hline Male & & & $49(17.9)$ \\
\hline Female & & & $219(81.7)$ \\
\hline \multicolumn{4}{|l|}{ Level of education } \\
\hline Diploma & & & $60(21.8)$ \\
\hline BSN & & & $196(72.3)$ \\
\hline Post Graduate degree & & & $15(0.4)$ \\
\hline \multicolumn{4}{|l|}{$\begin{array}{l}\text { Area of work (Unit/ } \\
\text { Floor) }\end{array}$} \\
\hline Critical care & & & $88(818)$ \\
\hline Medical surgical & & & $189(68.2)$ \\
\hline
\end{tabular}


Figure 1. Scree plot using the 29 items that were retained after the first round of analysis




Table 2. The rotated matrix that shows item loading in the four factor solution.

\begin{tabular}{llllll}
\hline & \multicolumn{3}{c}{ Component } \\
\cline { 2 - 5 } Item & \multicolumn{1}{c}{4} & 2 & 3 & 4 \\
\hline
\end{tabular}

There is no need to conduct PE if the patient will undergo diagnostic examination such as CT or MRI

.748

PE can be risky to some patients and may produce complications

.702

Conducting PE is NOT helpful because it is NOT specific test (able to identify normal findings when there is no problem)(demonstrate normal finding when there is no disorder)

PE can be a source of risk to the nurse

Conducting PE is NOT helpful because it is NOT sensitive test (able to identify abnormal findings when there is a problem) (demonstrate abnormality of finding when there is a disorder)

Sometime in the future, PE as we know it will NOT be that helpful

PE can be substituted by diagnostic procedures such as X-r, CT scan, and others

PE enhances communication and caring opportunities with the patient

$\mathrm{PE}$ is important to establish rapport and trusting relationship with the patient

PEis an integral part of nursing care for the patient .791

There are many diagnoses that can readily be made by the PE .669

Basing the choice of diagnostic studies on the results of PE is reliable way to limit unnecessary testing

I do NOT do PE because most of the nurses do NOT do PE anyways

I do NOT do PE often because I am NOT skillful or qualified

I do NOT do PE often because my PE skills had become poor

Many of the PE skills I learned seem unpractical

Many of the physical assessment skills are never or rarely used in practice.

.589

Performing PE on patient of opposite gender is stressful to me

I skip PE if the patient is from the opposite gender

Culture and norms can be obstacle to do physical exam specially when working

withpatient of opposite gender Extraction Method: Principal Component Analysis. Rotation Method: Varimax with Kaiser Normalization. a. Rotation converged in 8 iterations. 
Table 3. Factors that composed the scale

\begin{tabular}{|c|c|c|}
\hline Factor name & Items composing the factor & $\begin{array}{l}\text { Cronbach's alpha } \\
\text { for the } \\
\text { subscale/factor }\end{array}$ \\
\hline $\begin{array}{l}\text { Technical } \\
\text { deficiencies in PE }\end{array}$ & $\begin{array}{l}\text { 1. There is no need to conduct PE if the patient will } \\
\text { undergo diagnostic examination such as CT or MRI } \\
2 \text {. PE can be risky to some patients and may produce } \\
\text { complications } \\
3 \text {. Conducting PE is NOT helpful because it is NOT } \\
\text { specific test (able to identify normal findings when there is no } \\
\text { problem)(demonstrate normal finding when there is no disorder) } \\
4 . \quad \text { PE can be a source of risk to the nurse } \\
5 . \quad \text { Conducting PE is NOT helpful because it is NOT } \\
\text { sensitive test (able to identify abnormal findings when there is a } \\
\text { problem) (demonstrate abnormality of finding when there is a } \\
\text { disorder) } \\
6 \text {. Sometime in the future, PE as we know it will NOT be } \\
\text { that helpful } \\
7 . \quad \text { PE can be substituted by diagnostic procedures such as } \\
\text { x-r, CT scan, and others }\end{array}$ & .84 \\
\hline $\begin{array}{l}\text { Benefits and } \\
\text { usefulness of PE }\end{array}$ & $\begin{array}{l}\text { 1. PE enhances communication and caring opportunities } \\
\text { with the patient } \\
2 . \quad \text { PE is important to establish rapport and trusting } \\
\text { relationship with the patient } \\
3 . \quad \text { PE is an integral part of nursing care for the patient } \\
4 . \quad \text { There are many diagnoses that can readily be made by } \\
\text { the PE } \\
5 . \quad \text { Basing the choice of diagnostic studies on the results of } \\
\text { PE is reliable way to limit unnecessary testing }\end{array}$ & .813 \\
\hline $\begin{array}{l}\text { Barriers to conduct } \\
\text { PE }\end{array}$ & $\begin{array}{l}\text { 1. I do NOT do PE because most of the nurses do NOT do } \\
\text { PE anyways } \\
2 . \quad \text { I do NOT do PE often because I am NOT skillful or } \\
\text { qualified } \\
3 . \quad \text { I do NOT do PE often because my PE skills had become } \\
\text { poor } \\
\text { 4. Many of the PE skills I learned seem unpractical } \\
5 . \quad \text { Many of the physical assessment skills are never or } \\
\text { rarely used in practice. }\end{array}$ & .718 \\
\hline $\begin{array}{l}\text { Cultural } \\
\text { considerations }\end{array}$ & $\begin{array}{l}\text { 1. } \\
\text { to me } \\
2 . \\
\text { I. }\end{array}$ & .685 \\
\hline
\end{tabular}


Table 4. Descriptive statistics of the 20 items that composed the final version of the scale.

\begin{tabular}{|c|c|c|c|c|c|c|c|}
\hline \multirow[b]{2}{*}{ Item } & \multirow[t]{2}{*}{ Mean } & \multirow[t]{2}{*}{ Mode } & \multirow[t]{2}{*}{ SD } & \multirow[t]{2}{*}{ Range } & \multicolumn{3}{|c|}{ Percentiles } \\
\hline & & & & & 25 & 50 & 75 \\
\hline $\begin{array}{l}\text { I do NOT do PE because most of the nurses do NOT do } \\
\text { PE anyways }\end{array}$ & 2.67 & 2.0 & 1.23 & $1-5$ & 2.0 & 3.0 & 4.0 \\
\hline $\begin{array}{l}\text { I do NOT do PE often because I am NOT skillful or } \\
\text { qualified }\end{array}$ & 2.58 & 2.0 & 1.15 & $1-5$ & 2.0 & 2.0 & 3.0 \\
\hline $\begin{array}{l}\text { I do NOT do PE often because my PE skills had become } \\
\text { poor }\end{array}$ & 2.68 & 2.0 & 1.17 & $1-5$ & 2.0 & 2.0 & 4.0 \\
\hline Many of the PE skills I learned seem unpractical & 2.98 & 2.0 & 1.05 & $1-5$ & 2.0 & 3.0 & 4.0 \\
\hline $\begin{array}{l}\text { Many of the physical assessment skills are never or rarely } \\
\text { used in practice. }\end{array}$ & 3.30 & 4.0 & 1.02 & $1-5$ & 3.0 & 3.0 & 4.0 \\
\hline $\begin{array}{l}\text { PE can be risky to some patients and may produce } \\
\text { complications }\end{array}$ & 2.64 & 2.0 & 1.11 & $1-5$ & 2.0 & 2.0 & 4.0 \\
\hline PE can be a source of risk to the nurse & 2.79 & 2.0 & 1.21 & $1-5$ & 2.0 & 3.0 & 4.0 \\
\hline PEis an integral part of nursing care for the patient & 3.72 & 4.0 & .98 & $1-5$ & 3.0 & 4.0 & 4.0 \\
\hline $\begin{array}{l}\mathrm{PE} \text { is important to establish rapport and trusting } \\
\text { relationship with the patient }\end{array}$ & 3.68 & 4.0 & .93 & $1-5$ & 3.0 & 4.0 & 4.0 \\
\hline $\begin{array}{l}\text { PE enhances communication and caring opportunities } \\
\text { with the patient }\end{array}$ & 3.82 & 4.0 & .85 & $1-5$ & 4.0 & 4.0 & 4.0 \\
\hline $\begin{array}{l}\text { There are many diagnoses that can readily be made by the } \\
\text { PE }\end{array}$ & 3.57 & 4.0 & .95 & $1-5$ & 3.0 & 4.0 & 4.0 \\
\hline $\begin{array}{l}\text { Basing the choice of diagnostic studies on the results of } \\
\text { PE is reliable way to limit unnecessary testing }\end{array}$ & 3.66 & 4.0 & .89 & $1-5$ & 3.0 & 4.0 & 4.0 \\
\hline $\begin{array}{l}\text { PE can be substituted by diagnostic procedures such as X- } \\
\text { r, CT scan, and others }\end{array}$ & 2.75 & 2.0 & 1.08 & $1-5$ & 2.0 & 3.0 & 4.0 \\
\hline $\begin{array}{l}\text { There is no need to conduct PE if the patient will undergo } \\
\text { diagnostic examination such as CT or MRI }\end{array}$ & 2.64 & 2.0 & .99 & $1-5$ & 2.0 & 2.0 & 3.0 \\
\hline $\begin{array}{l}\text { Sometime in the future, PE as we know it will NOT be } \\
\text { that helpful }\end{array}$ & 2.79 & 2.0 & 1.08 & $1-5$ & 2.0 & 3.0 & 4.0 \\
\hline I skip PE if the patient is from the opposite gender & 3.21 & 4.0 & 1.09 & $1-5$ & 2.0 & 3.0 & 4.0 \\
\hline $\begin{array}{l}\text { Performing PE on patient of opposite gender is stressful } \\
\text { to me }\end{array}$ & 3.46 & 4.0 & 1.05 & $1-5$ & 3.0 & 4.0 & 4.0 \\
\hline $\begin{array}{l}\text { Conducting PE is NOT helpful because it is NOT } \\
\text { sensitive test (able to identify abnormal findings when } \\
\text { there is a problem) (demonstrate abnormality of finding } \\
\text { when there is a disorder) }\end{array}$ & 2.67 & 2.0 & 1.08 & $1-5$ & 2.0 & 2.0 & 4.0 \\
\hline $\begin{array}{l}\text { Conducting PE is NOT helpful because it is NOT specific } \\
\text { test (able to identify normal findings when there is no } \\
\text { problem)(demonstrate normal finding when there is no } \\
\text { disorder) }\end{array}$ & 2.67 & 2.0 & 1.01 & $1-5$ & 2.0 & 3.0 & 3.0 \\
\hline $\begin{array}{l}\text { Culture and norms can be obstacle to do PE specially } \\
\text { when working with patient of opposite gender }\end{array}$ & 3.48 & 4.0 & .996 & $1-5$ & 3.0 & 4.0 & 4.0 \\
\hline
\end{tabular}


Appendix 1. The 55 items from which he final scale was derived.

\begin{tabular}{|c|c|c|c|c|c|c|}
\hline \# & Item & 1 & 2 & 3 & 4 & 5 \\
\hline 1. & I often do physical exam & & & & & \\
\hline 2. & Doing both comprehensive and focused exam is unneeded & & & & & \\
\hline 3. & Conducting physical exam is mainly the responsibility of the physician & & & & & \\
\hline 4. & Even physicians do NOT often do physical exam & & & & & \\
\hline 5. & I do NOT do physical exam if the physicians already did it & & & & & \\
\hline 6. & I do NOT do physical exam because most of the nurses do NOT do physical exam anyways & & & & & \\
\hline 7. & In general, nurses do NOT do physical exam as often as they should & & & & & \\
\hline 8. & Conducting physical exam is fundamental to nurses and should always be done & & & & & \\
\hline 9. & I do NOT do physical exam often because I am NOT skillful or qualified & & & & & \\
\hline 10. & $\begin{array}{l}\text { Only specialized nurses such as critical care nurses, clinical nurse specialists, and nurse } \\
\text { practitioners can perform proper and complete physical exam }\end{array}$ & & & & & \\
\hline 11. & General nurses are poorly qualified to do accurate examination & & & & & \\
\hline 12. & I do physical exam because it makes a difference in my patient care & & & & & \\
\hline 13. & There is a discrepancy between what is taught and what is practiced regarding physical exam & & & & & \\
\hline 14. & Conducting physical exam does NOT affect patient outcome & & & & & \\
\hline 15. & In my opinion, general nurses are NOT as efficient as doctors in doing physical exam & & & & & \\
\hline 16. & I do NOT do physical exam often because my physical exam skills had become poor & & & & & \\
\hline 17. & The finding of the physical exam depends on the level of expertise of one who conduct it & & & & & \\
\hline 18. & Even experience does NOT necessarily mean accurate physical exam findings & & & & & \\
\hline 19. & I do NOT do physical exam often because I did NOT learn the skills properly. & & & & & \\
\hline 20. & I do physical exam because it increases my confidence in my own ability & & & & & \\
\hline 21. & I do NOT do physical exam because no one takes my findings seriously & & & & & \\
\hline 22. & Physical exam requires preparing many special tools & & & & & \\
\hline 23 & Conducting PE takes long time & & & & & \\
\hline 24. & $\begin{array}{l}\text { The high pressure of work makes doing comprehensive and focused physical exam almost } \\
\text { impossible }\end{array}$ & & & & & \\
\hline 25. & Many of the physical exam skills I learned seem unpractical & & & & & \\
\hline 26. & Physical exam is useful only when patient is exhibiting classic symptoms. & & & & & \\
\hline 27. & Doing physical exam is necessary only for certain hospital units but NOT all & & & & & \\
\hline 28. & Many of the physical assessment skills are never or rarely used in practice. & & & & & \\
\hline 29. & $\begin{array}{l}\text { Conducting physical exam is NOT helpful because it is NOT sensitive test (able to identify } \\
\text { abnormal findings when there is a problem) (demonstrate abnormality of finding when there is a } \\
\text { disorder) }\end{array}$ & & & & & \\
\hline 30. & $\begin{array}{l}\text { Conducting physical exam is NOT helpful because it is NOT specific test (able to identify } \\
\text { normal findings when there is no problem)(demonstrate normal finding when there is no } \\
\text { disorder) }\end{array}$ & & & & & \\
\hline 31. & Patient history and the interview can be sufficient to identify the needed diagnostic tests & & & & & \\
\hline 32. & The detected physical exam findings are no guarantee of the truth of these results & & & & & \\
\hline 33. & physical exam can be risky to some patients and may produce complications & & & & & \\
\hline 34. & physical exam can be a source of risk to the nurse & & & & & \\
\hline 35. & PE is an integral part of nursing care for the patient & & & & & \\
\hline 36. & Physical exam is important to establish rapport and trusting relationship with the patient & & & & & \\
\hline 37. & Physical exam enhances communication and caring opportunities with the patient & & & & & \\
\hline 38. & There are many diagnoses that can readily be made by the physical exam & & & & & \\
\hline 39. & $\begin{array}{l}\text { Basing the choice of diagnostic studies on the results of physical exam is reliable way to limit } \\
\text { unnecessary testing }\end{array}$ & & & & & \\
\hline 40. & There is no agreement on the way physical exam skills are conducted/done & & & & & \\
\hline 41. & There is no agreement on the way physical exam results are interpreted & & & & & \\
\hline 42. & $\begin{array}{l}\text { Physical exam is important because it assessed person's unique psychological, social, and } \\
\text { physiological response to his condition }\end{array}$ & & & & & \\
\hline 43. & Physical exam is more useful in detecting deterioration in condition than in making diagnosis & & & & & \\
\hline
\end{tabular}


Page 27 of 27

Nursing Forum

27

1

2

3

4

5

6

7

8

9

10

11

12

13

14

15

16

17

18

19

20

21

22

23

24

25

26

27

28

29

30

31

32

33

34

35

36

37

38

39

40

41

42

43

44

45

46

47

48

49

50

51

52

53

54

55

56

57

58

59

60

\begin{tabular}{|l|l|l|l|l|l|}
\hline 44. & I learn physical exam skills only because they are required in class & & & \\
\hline 45. & I do physical exam only because it is required by my institution & & & \\
\hline 46. & Diagnostic tests are more efficient than physical exam in detecting patient problem. & & & & \\
\hline 47. & $\begin{array}{l}\text { Relying primarily on imaging studies without considering physical exam can lead to serious } \\
\text { mistakes }\end{array}$ & & & & \\
\hline 48. & physical exam can be substituted by diagnostic procedures such as x-r, CT scan, and others & & & & \\
\hline 49. & $\begin{array}{l}\text { There is no need to conduct physical exam if the patient will undergo diagnostic exam such as } \\
\text { CT or MRI }\end{array}$ & & & \\
\hline 50. & $\begin{array}{l}\text { New machines such as dinamap, patient monitor, pulse oxymetry, Doppler U/S can provide the } \\
\text { nurse with all the data the nurses need }\end{array}$ & & & \\
\hline 51. & Sometime in the future, physical exam as we know it will NOT be that helpful & & & \\
\hline 52. & I skip PE if the patient is from the opposite gender & & & \\
\hline 53. & Culture and norms can be obstacle to do physical exam & & & \\
\hline 54. & Performing physical exam on patient of opposite gender is stressful to me & & \\
\hline 55. & Performing physical exam on patient of opposite gender is stressful to the patient & & & \\
\hline
\end{tabular}

